\title{
Optimal portfolio strategies under a shortfall constraint
}

\author{
Daniel Akume* $\quad$ Bernd Luderer ${ }^{\dagger} \quad$ Ralf Wunderlich ${ }^{\ddagger}$
}

Received: 12 November 2008; Revised: 26 February 2009; Accepted: 10 March 2009

\begin{abstract}
We impose dynamically, a shortfall constraint in terms of Tail Conditional Expectation on the portfolio selection problem in continuous time, in order to obtain optimal strategies. The financial market is assumed to comprise $n$ risky assets driven by geometric Brownian motion and one risk-free asset. The method of Lagrange multipliers is combined with the Hamilton-Jacobi-Bellman equation to insert the constraint into the resolution framework. The constraint is re-calculated at short intervals of time throughout the investment horizon. A numerical method is applied to obtain an approximate solution to the problem. It is found that the imposition of the constraint curbs investment in the risky assets.
\end{abstract}

Key words: Portfolio optimization, value-at-risk, quadratic utility, tail conditional expectation.

\section{Introduction}

In recent years, particular stress has been laid on the substitution of variance as a risk measure in the standard Markowitz [11] mean-variance problem. Since it makes no distinction between positive and negative deviations from the mean, variance is a good measure of risk only for distributions that are (approximately) symmetric around the mean, such as the normal distribution or, more generally, elliptical distributions [12]. However, in most cases, such as in portfolios containing options, one deals with wealth distributions that are highly skewed. It is thus more reasonable to consider asymmetric risk measures since individuals are typically loss averse. In this regard, Value-at-Risk (VaR), a downside risk measure, has emerged as an industry standard with regulatory authorities, such as the Basle Committee on Banking Supervision which enforces its use [9].

Despite its widespread acceptance, VaR is known to possess unappealing features. Artzner et al. [3] proposed an axiomatic foundation for risk measures, by identifying four properties that a reasonable risk measure should satisfy. They also provided a characterization of

\footnotetext{
${ }^{*}$ Corresponding author: Computer Science Department, University of Buea, P.O. Box 63, Buea, Cameroon, email: d_akume@yahoo.ca

${ }^{\dagger}$ Faculty of Mathematics, Chemnitz University of Technology, P.O. Box 964, Chemnitz, 09107, Germany.

${ }^{\ddagger}$ Mathematics Department, Zwickau University of Applied Sciences, P.O. Box 201037, Zwickau, 08012, Germany.
} 
the risk measures satisfying these properties, which they called coherent risk measures. By these axioms, VaR is not coherent. Tail Conditional Expectation (TCE), on the other hand, is a coherent risk measure for an underlying continuous distribution [14].

The focus in this paper is the dynamic portfolio choice of a trader subject to a risk limit specified in terms of TCE. We maximize the agent's utility ${ }^{1}$ over wealth throughout the investment horizon. The TCE constraint is re-calculated and re-imposed at every short interval of time, throughout the investment horizon. The portfolio is assumed constant over each such short interval (re-evaluation horizon), as is the case in practice. This art of dynamically re-evaluating the constraint has not yet received adequate attention in the existing literature. We show through numerical simulations, by applying an algorithm similar to that in Yiu [15], that the introduction of a TCE constraint causes investment in risky $\operatorname{assets}^{2}$ to be controlled. In our numerical experiments we use two risky assets, as opposed to just one, which is common in the existing literature.

The remainder of this paper is structured as follows. In Section 2, we model the financial market and describe the portfolio dynamic. In Section 3 we derive the Value-at-Risk and Tail Conditional Expectation formulas for the market model under study, while in Section 4 we make precise the optimal control problem to be solved. The solution of the problem is derived in Section 5 by using the Lagrangian technique to combine the Hamilton-JacobiBellman (HJB) equation and the TCE constraint. In Section 6, a numerical algorithm is presented to obtain an approximate solution to the TCE-constrained problem. Section 8 is devoted to the computation of the value function for use in the numerical procedure. We also present some simulation results with an ensuing discussion in Section 8, while Section 9 concludes the paper.

\section{The model}

We consider a standard Black-Scholes-type market (see Korn [10] for relevant definitions) consisting of one risk-free bond and $n$ risky stocks. The financial market is continuous-time with a finite time horizon $[0, T]$.

Uncertainty in the financial market is modelled by a probability space $(\Omega, \mathcal{F}, P)$, equipped with a filtration that is a non-decreasing family $\mathbb{F}=\left(\mathcal{F}_{t}\right)_{t \in[0, T]}$ of sub- $\boldsymbol{\sigma}$-fields $\mathcal{F}_{s} \subseteq \mathcal{F}_{t} \subseteq \mathcal{F}$, $0 \leq s<t<\infty$. It is assumed throughout this paper that all inequalities as well as equalities hold $\boldsymbol{P}$-almost surely. Moreover, it is assumed that all stated processes are well defined without giving any regularity conditions ensuring this. The risk-free rate $r=r_{t}$ of the risk-free asset (bond) $S^{0}$ is supposed to evolve according to $d S_{t}^{0}=r S_{t}^{0} d t, S_{0}^{0}=s$.

For the risky assets (stocks), whose prices are denoted by $\boldsymbol{S}_{t}=\left(S_{t}^{1}, \ldots, S_{t}^{n}\right)$ for some

\footnotetext{
${ }^{1}$ Here, we use a quadratic utility function. Akume and Mbakop [1] solve a similar problem with power utility.

${ }^{2}$ See Akume and Mbakop [1] for a similar problem combining both investment and consumption strategies.
} 
$n \in \mathbb{N}$, the basic evolution model is that of a log-normal diffusion process,

$$
\begin{aligned}
\frac{d S_{t}^{i}}{S_{t}^{i}} & =\mu^{i} d t+\sum_{j=1}^{k} \sigma^{i j} d W_{t}^{j}, & & t \in[0, T], \\
S_{0}^{i} & =s^{i}, & & i=1, \ldots, n,
\end{aligned}
$$

where, for some $k \in \mathbb{N}, \boldsymbol{W}_{t}=\left[W_{t}^{1}, \ldots, W_{t}^{k}\right]^{\prime}$, with the symbol $\left({ }^{\prime}\right)$ denoting a transpose, is a $k$-dimensional standard Wiener process, i.e. a vector of $k$ independent one-dimensional Wiener processes. The $n$-vector $\boldsymbol{\mu}=\boldsymbol{\mu}_{t}=\left(\mu_{t}^{1}, \ldots, \mu_{t}^{n}\right)^{\prime}$, contains the expected instantaneous rates of return and the $n \times k$-matrix $\boldsymbol{\sigma}=\boldsymbol{\sigma}_{t}=\left[\sigma_{t}^{i j}\right](i=1, \ldots, n, j=1, \ldots, k)$ measures the instantaneous sensitivities of the risky asset prices with respect to exogenous shocks so that the $(n \times n)$-matrix $\boldsymbol{\sigma} \boldsymbol{\sigma}^{\prime}$ contains the variance and covariance rates of instantaneous rates of return. Here $\boldsymbol{\mu}$ and $\boldsymbol{\sigma}$ must be adapted to the information filtration $\mathbb{F}=\left(\mathcal{F}_{t}\right)$.

An agent invests according to an investment strategy that can be described by the $(n+1)$ dimensional, $\mathcal{F}_{t}$-predictable process $\boldsymbol{x}_{t}=\left(x_{t}^{0}, x_{t}^{1}, \ldots, x_{t}^{n}\right)^{\prime}$, where $x_{t}^{i}(i=1, \ldots, n)$ denotes the number of shares of asset $i$ held in the portfolio at time $t$ ( $i=0$ refers to the bond). The process $\boldsymbol{x}_{t}$ describes an investor's portfolio as carried forward through time. The value of the investor's wealth at time $t$ is then

$$
V_{t}^{x}=x_{t}^{0} S_{t}^{0}+\sum_{i=1}^{n} x_{t}^{i} S_{t}^{i},
$$

where $x_{t}^{i} S_{t}^{i}$ represents the amount invested in asset $i$ at time $t$. Equivalently, one may consider the vector $\boldsymbol{\theta}_{t}=\left(\theta_{t}^{1}, \ldots, \theta_{t}^{n}\right)^{\prime}$, where

$$
\theta_{t}^{i}=\frac{x_{t}^{i} S_{t}^{i}}{V_{t}^{x}}, \quad i=1, \ldots, n
$$

denotes the fraction of wealth invested in the risky asset $i$ at time $t$, whereby the remaining fraction $1-\sum_{j=1}^{n} \theta_{t}^{i}$ of the agent's wealth is invested in the risk-free asset. It is assumed that $\theta_{t}^{1}, \ldots, \theta_{t}^{n}$ are admissible and $\mathcal{F}_{t^{-}}$adapted control processes. That is, $\theta_{t}^{1}, \ldots, \theta_{t}^{n}$ are nonanticipative functions that satisfy the condition of bounded variation $\int_{0}^{T} \sum_{1=1}^{n}\left(\theta_{t}^{i}\right)^{2} d t<\infty$ for an investment time horizon $T<\infty$. The corresponding portfolio value process reads

$$
\begin{aligned}
d V_{t}^{\boldsymbol{\theta}} & =V_{t}^{\boldsymbol{\theta}}\left[\left(1-\sum_{i=1}^{n} \theta_{t}^{i}\right) \frac{d S_{t}^{0}}{S_{t}^{0}}+\sum_{i=1}^{n} \theta_{t}^{i} \frac{d S_{t}^{i}}{S_{t}^{i}}\right] \\
& =V_{t}^{\boldsymbol{\theta}}\left[\left(r+\sum_{i=1}^{n} \theta_{t}^{i}\left(\mu^{i}-r\right)\right) d t+\sum_{i=1}^{n} \sum_{j=1}^{k} \theta_{t}^{i} \sigma^{i, j} d W_{t}^{j}\right], t \in[0, T], \\
V_{0}^{\boldsymbol{\theta}} & =v .
\end{aligned}
$$

Let $\boldsymbol{\mu}-\boldsymbol{r}=\left[\mu_{t}^{1}-r, \ldots, \mu_{t}^{n}-r\right]^{\prime}$. Then equation (1) can be rewritten more succinctly in matrix notation as

$$
d V_{t}^{\boldsymbol{\theta}}=V_{t}^{\boldsymbol{\theta}}\left[\left(r_{t}+\boldsymbol{\theta}_{t}^{\prime}\left(\boldsymbol{\mu}_{t}-\boldsymbol{r}\right)\right) d t+\boldsymbol{\theta}_{t}^{\prime} \boldsymbol{\sigma}_{t} d \boldsymbol{W}_{t}\right], V_{0}^{\boldsymbol{\theta}}=v .
$$


Thus,

$$
V_{t}^{\boldsymbol{\theta}}=\left(1-\sum_{i=1}^{n} \theta_{t}^{i}\right) V_{t}^{\boldsymbol{\theta}}+\sum_{i=1}^{n} \theta_{t}^{i} V_{t}^{\boldsymbol{\theta}}
$$

We have adopted the incomplete market asset pricing setting of He and Pearson [7]. To eliminate redundant assets, we assume that $\boldsymbol{\sigma}$ is of full row rank — that is, $\boldsymbol{\sigma} \boldsymbol{\sigma}^{\prime}$ is an invertible matrix.

\section{Tail conditional expectation}

Here we start by defining Value-at-Risk since the subsequent definition of Tail Conditional Expectation (TCE) will depend on it.

\section{Definition 1 (Value-at-Risk)}

Given some probability level $\alpha \in(0,1)$, a time $t$ wealth benchmark $\Upsilon_{t}$ and horizon $\Delta t$, the Value-at-Risk of time $t$ wealth $V_{t}$ at the confidence level $(1-\alpha)$, denoted VaR $R_{t}^{\alpha}$, is given by the smallest number $L$ for which the probability that the loss $G_{t+\Delta t}:=\Upsilon_{t+\Delta t}-V_{t+\Delta t}^{\theta}$ exceeds $L$ is no larger than $\alpha$. That is, $\operatorname{VaR}_{t}^{\alpha}=\inf \left\{L \geq 0: \boldsymbol{P}\left(G_{t+\Delta t} \geq L \mid \mathcal{F}_{t}\right) \leq \alpha\right\}:=\left(Q_{t}^{\alpha}\right)^{-}$, where $Q_{t}^{\alpha}=\sup \left\{L \in \mathbb{R}: \boldsymbol{P}\left(\left(V_{t+\Delta t}^{\theta}-\Upsilon_{t+\Delta t}\right) \leq L \mid \mathcal{F}_{t}\right) \leq \alpha\right\}$ is the quantile of the projected wealth surplus at the horizon $t+\Delta t$ and $x^{-}=\max [0,-x]$.

Thus, $\operatorname{VaR}_{t}^{\alpha}=0$ for $Q_{t}^{\alpha} \geq 0 . \quad V a R_{t}^{\alpha}$ is therefore the loss of wealth with respect to a benchmark $\Upsilon_{t+\Delta t}$ at the horizon $\Delta t$ which could be exceeded only with a small conditional probability $\alpha$ if the current portfolio $\boldsymbol{\theta}_{t}$ were kept unchanged. Typical values for the probability level $\alpha$ are $\alpha=0.05$ or $\alpha=0.01$. In market risk management the time horizon $\Delta t$ is usually one or ten days.

\section{Proposition 1 (Computation of Value-at-Risk)}

It holds that

$$
V a R_{t}^{\alpha}=\left(V_{t}^{\theta} \exp \left[\Phi^{-1}(\alpha)\left\|\boldsymbol{\theta}_{t}^{\prime} \boldsymbol{\sigma}\right\| \sqrt{\Delta t}+\left(\boldsymbol{\theta}_{t}^{\prime}(\boldsymbol{\mu}-\boldsymbol{r})+r-\frac{1}{2}\left\|\boldsymbol{\theta}_{t}^{\prime} \boldsymbol{\sigma}\right\|^{2}\right) \Delta t\right]-\Upsilon_{t+\Delta t}\right)^{-}
$$

where $\Phi(\cdot)$ and $\Phi^{-1}(\cdot)$ denote the normal distribution and inverse normal distribution functions respectively, and where $\|\cdot\|$ is the Euclidean norm.

For a proof of this proposition, see Akume and Mbakop [1]. Tail Conditional Expectation is closely related to the concept of Value-at-Risk, but overcomes some of the conceptual deficiencies of Value-at-Risk [14]. In particular, it is a coherent risk measure [2].

\section{Definition 2 (Tail Conditional Expectation)}

Consider the loss distribution $G_{t+\Delta t}:=\Upsilon_{t+\Delta t}-V_{t+\Delta t}^{\theta}$ represented by a continuous distribution function $F_{G_{t+\Delta t}}$ with $\int_{\mathbb{R}}\left|G_{t+\Delta t}\right| d F\left(G_{t+\Delta t}\right)<\infty$. Then the TCE $E_{t}^{\alpha}$ at confidence level $(1-\alpha)$ is defined as $T C E_{t}^{\alpha}=\mathbb{E}_{t}\left\{G_{t+\Delta t} \mid G_{t+\Delta t} \geq V a R_{t}^{\alpha}\right\}$. 
The Tail Conditional Expectation of wealth $V_{t}$ at time $t$ is therefore the conditional expected value of the loss exceeding $V a R_{t}^{\alpha}$. Again, given the log-normal distribution of asset returns, the $T C E_{t}^{\alpha}$ may be computed explicitly as can be seen in the following proposition.

\section{Proposition 2 (Computation of Tail Conditional Expectation)}

It holds that

$$
T C E_{t}^{\alpha}=\frac{\alpha \Upsilon_{t+\Delta t}-V_{t}\left[\exp \left(\left(\boldsymbol{\theta}_{t}^{\prime}(\boldsymbol{\mu}-\boldsymbol{r})+r\right) \Delta t\right) \Phi\left(\Phi^{-1}(\alpha)-\left\|\boldsymbol{\theta}_{t}^{\prime} \boldsymbol{\sigma}\right\| \sqrt{\Delta t}\right)\right]}{\alpha},
$$

where $\Phi(\cdot)$ and $\Phi^{-1}(\cdot)$ denote the normal distribution and inverse normal distribution functions, respectively.

For a proof of this proposition, again see Akume and Mbakop [1].

\section{Statement of the problem}

We seek the optimal asset allocation that maximizes (over all allowable $\boldsymbol{\theta}_{t}$ ) the expected utility of discounted wealth over the entire horizon $[0, T]$, for a risk-averse investor who limits his risk by imposing an upper bound on the TCE.

The choice of this problem is motivated by the income drawdown option in defined contribution pension schemes. Such an option allows the member who retires not to convert the accumulated capital into annuity immediately at retirement, but to defer the purchase of the annuity until a certain point in time after retirement. The period of time can be limited to time $T$. Usually, freedom is given for a fixed number of years after retirement and at a certain age the annuity is bought.

Here, we consider the income drawdown option and investigate, by means of stochastic optimal control techniques, what should be the optimal investment and consumption allocation of the fund after retirement until the purchase of the annuity. The reason the pensioner chooses the drawdown option is the hope of being able to invest the accumulated capital at retirement and increase its value in order to buy a better annuity in the future than the one he otherwise could have bought at retirement.

Thus, our objective is to maximize the expected utility of wealth from retirement until the interruption of the income drawdown option. Therefore, in mathematical terms the final optimal control problem with TCE constraint is

$$
\max _{\{\theta\} \in A(v)} \mathbb{E}_{0, V_{0}}\left\{\int_{0}^{T} e^{-\rho s} U^{1}\left(V_{s}, s\right) d s+e^{-\rho T} U^{2}\left(V_{T}, T\right)\right\}
$$

subject to the wealth dynamic

$$
d V_{t}^{\theta}=\left[V_{t}^{\theta}\left(\boldsymbol{\theta}_{t}^{\prime}(\boldsymbol{\mu}-\boldsymbol{r})+r\right)\right] d t+V_{t}^{\theta} \boldsymbol{\theta}_{\boldsymbol{t}}{ }^{\prime} \boldsymbol{\sigma} d \boldsymbol{W}_{t}, V_{0}^{\theta}=v
$$

and the TCE constraint

$$
\frac{1}{\alpha}\left(\alpha \Upsilon_{t+\Delta t}-V_{t}^{\theta}\left[\exp \left(\left(\boldsymbol{\theta}_{t}^{\prime}(\boldsymbol{\mu}-\boldsymbol{r})+r\right) \Delta t\right) \cdot \Phi\left(\Phi^{-1}(\alpha)-\left\|\boldsymbol{\theta}_{t}^{\prime} \boldsymbol{\sigma}\right\| \sqrt{\Delta t}\right)\right]\right) \leq \varepsilon(v, t)
$$


for all $t \in[0, T)$, where $\mathbb{E}_{t, v}$ denotes the expectation operator at time $t$, given $V_{t}^{\theta}=v$ (and given the chosen investment strategies), where $U^{1}$ and $U^{2}$ are twice differentiable, concave utility functions, where $\varepsilon(v, t)$ is an upper bound on TCE and where $\rho>0$ is the rate at which wealth is discounted. Note that we allow room for the running utility function to differ (be weighted differently) from the terminal utility function. Let $U^{1}(x)=U^{2}(x)=U(x)=K x-(1-K)(x-\psi)^{2}$ denote a quadratic utility which privileges with a weight $K \in(0,1)$ a large wealth, whereas it penalizes with a weight $(1-K)$ the square of the spread between the current wealth $x$ and a target wealth, denoted $\psi$. Quadratic utilities are widely used in the literature [6].

\section{Optimality conditions}

We adapt a dynamic programming approach to solve the HJB equation associated with the utility maximization problem (2). Following Fleming and Rishel [5], the corresponding HJB equation is given by

$$
J_{t}(v, t)-\rho J(v, t)+U(v)+\sup _{\theta_{t}}\left\{D^{\theta_{t}} J(v, t)\right\}=0
$$

where $t \in[0, T]$ is the horizon, where $V_{t}^{\theta}=v$ is any admissible state and where

$$
D^{\theta_{t}} J(v, t)=J_{v}(v, t)\left(v\left[\theta_{t}^{\prime}(\mu-r)+r\right]\right)+\frac{1}{2} v^{2}\left\|\theta_{t}^{\prime} \sigma\right\|^{2} J_{v v}(v, t)
$$

subject to the terminal condition $J(v, T)=U(v)$, where the value function is given by

$$
J(v, t)=\sup _{\{\theta\} \in A(v)} \mathbb{E}_{t, V_{t}}\left\{\int_{t}^{T} e^{-\rho(s-t)} U\left(V_{s}, s\right) d s+e^{-\rho(T-t)} U\left(V_{T}, T\right)\right\}
$$

with subscripts on $J$ denoting partial derivatives and where $V_{t}^{\theta}=v$ denotes the wealth realization at time $t$.

In solving the HJB equation (4), the static optimization problem

$$
\max _{\theta_{t}}\left\{D^{\theta_{t}} J(v, t)\right\}
$$

subject to the TCE constraint (3), may be tackled separately to reduce the HJB equation (4) to a nonlinear partial differential equation of $J$ only.

Introducing the Lagrangian function

$$
\begin{aligned}
& \mathcal{L}\left(\theta_{t}(v, t), \lambda(v, t)\right)=J_{v}(v, t)\left(v\left[\theta_{t}^{\prime}(\mu-r)+r\right]\right)+\frac{1}{2} v^{2}\left\|\theta_{t}^{\prime} \sigma\right\|^{2} J_{v v}(v, t)+U(v)-\{\lambda(v, t) \\
& \left.\times\left(\alpha \Upsilon_{t+\Delta t}-v\left[\exp \left(\left(\theta_{t}^{\prime}(\mu-r)+r\right) \Delta t\right) \cdot \Phi\left(\Phi^{-1}(\alpha)-\left\|\theta_{t}^{\prime} \sigma\right\| \sqrt{\Delta t}\right)\right]-\varepsilon_{1}(v, t)\right)\right\},
\end{aligned}
$$


where $\lambda$ is the Lagrange multiplier and $\varepsilon_{1}=\varepsilon \cdot \alpha$, the first-order necessary conditions with respect to $\theta$ and $\lambda$ respectively, of the static optimization problem (5), are given by

$$
\begin{aligned}
& v J_{v}(\mu-r)+ \frac{1}{2} J_{v v} v^{2} \sigma \sigma^{\prime} \theta_{t}+\lambda(v, t) v\left((\mu-r) \Delta t \exp \left(\left(\theta_{t}^{\prime}(\mu-r)+r\right) \Delta t\right)\right. \\
& \cdot \Phi\left(\Phi^{-1}(\alpha)-\left\|\theta_{t}^{\prime} \sigma\right\| \sqrt{\Delta t}\right)-\exp \left(\left(\theta_{t}^{\prime}(\mu-r)+r\right) \Delta t\right) \cdot \frac{\sqrt{\Delta t}}{2} \frac{\sigma \sigma^{\prime} \theta_{t}}{\left\|\theta_{t}^{\prime} \sigma\right\|} \\
&\left.\cdot \frac{1}{\sqrt{2 \pi}} \exp \left[-\frac{1}{2}\left(\Phi^{-1}(\alpha)-\left\|\theta_{t}^{\prime} \sigma\right\| \sqrt{\Delta t}\right)^{2}\right]\right)=0,
\end{aligned}
$$

where $\pi \approx 3.14159$ (we have applied the product law of differentiation and the fundamental theorem of calculus in deriving the latter first-order derivative), and

$$
\left.H(v, t)=\alpha \Upsilon_{t+\Delta t}+v\left[\exp \left(\left(\theta_{t}^{\prime}(\mu-r)+r\right) \Delta t\right) \cdot \Phi\left(\Phi^{-1} \alpha\right)-\left\|\theta_{t}^{\prime} \sigma\right\| \sqrt{\Delta t}\right)\right]+\varepsilon_{1}(v, t)=0,
$$

while the complimentary slackness conditions are $\lambda(v, t) H(v, t)=0$ and $\lambda(v, t) \geq 0$.

Simultaneous resolution of these first-order conditions yields the optimal solutions $\boldsymbol{\theta}^{\text {opt }}$ and $\lambda^{\text {opt }}$. Substituting these into (4) gives the partial differential equation

$$
\begin{aligned}
-\rho J(v, t)+ & J_{t}(v, t)+J_{v}(v, t)\left(v\left[\left(\boldsymbol{\theta}^{o p t}(v, t)\right)^{\prime}(\boldsymbol{\mu}-\mathbf{r})+r\right]\right) \\
& +K v-(1-K)(v-\psi)^{2}+\frac{1}{2} J_{v v}(v, t) v^{2}\left(\boldsymbol{\theta}^{o p t}(v, t)\right)^{\prime} \boldsymbol{\sigma} \boldsymbol{\sigma}^{\prime}\left(\boldsymbol{\theta}^{o p t}(v, t)\right)=0,
\end{aligned}
$$

with terminal condition $J(v, T)=K v-(1-K)(v-\psi)^{2}$, which may then be solved for the optimal value function $J^{o p t}(v, t)$.

Due to the nonlinearity of $\boldsymbol{\theta}^{\text {opt }}$, the first-order conditions together with the HJB equation are a non-linear system. Hence the differential equation (6) has no analytic solution and numerical methods such as Newton's method or Sequential Quadratic Programming (SQP) (see, for example, Nocedal and Wright [13]) are required to solve for $\boldsymbol{\theta}^{\text {opt }}(v, t), \lambda^{\text {opt }}(v, t)$ and $J^{o p t}(v, t)$ iteratively.

\section{Numerical solution}

We use an iterative algorithm similar to that of Yiu [15] which yields a $\mathcal{C}^{2,1}$ approximation $\widehat{J}$ of the exact solution $J$ and $\widehat{\theta}_{t}$, the investment strategy related to $\widehat{J}$.

When the optimal solution strictly satisfies the TCE constraint (3), the Lagrange multiplier $\lambda(v, t)$ is zero. If the constraint is active, the multiplier is positive.

First, we divide the domain of resolution into a grid of $n_{v} \times n_{t}$ mesh points. Iterations are indexed by $k$.

1. For each point $(t, v)$, with $t \in\left\{0, \Delta t, \ldots, n_{t} \Delta t\right\}$ and $v \in\left\{0, \Delta v, \ldots, n_{v} \Delta v\right\}$, we compute the value function $\widehat{J}^{k=0}=J(v, t)$ and the optimal strategy $\boldsymbol{\theta}_{t}^{\text {opt }}$ of the unconstrained problem. All Lagrange multipliers are set to zero, i.e. $\lambda_{t, v}^{k=0}=0$. This solution is the starting point of the algorithm. 
2. For all points of the grid, the constraint is checked. If the constraint is not active (i.e. $T C E_{t}^{\alpha}<\varepsilon(v, t)$ ), the multiplier is zero (i.e. $\lambda_{t, v}^{k+1}=0$ ) and

$$
\boldsymbol{\theta}_{t}^{k+1}=-\frac{\widehat{J}_{v}}{v \widehat{J}_{v v}}(\boldsymbol{\mu}-\boldsymbol{r})\left(\boldsymbol{\sigma}^{\prime} \boldsymbol{\sigma}\right)^{-1}
$$

is the solution of a similar equation to that of the unconstrained case.

If the $T C E_{t}^{\alpha}$ constraint is active, (i.e. $T C E_{t}^{\alpha} \geq \varepsilon$ ), we solve a nonlinear system in $\lambda_{t, v}^{k+1}$ and $\widehat{\theta}_{t}^{j+1}$. This nonlinear system comprises of the first-order necessary conditions of the static optimization problem (5). That system is numerically solved by the sequential quadratic programming method (see Nocedal and Wright [13]).

3. The last stage consists of the calculation of the value function $\widehat{J}^{k+1}$ according to the investment strategy $\widehat{\theta}_{t}^{k+1}$ as detailed in the following section.

4. Increment $k$ (i.e. $k \leftarrow k+1$ ) and return to step 2 until the error at time $t$ from wealth level $v, \epsilon_{t, v}$, satisfies $\left|\epsilon_{t, v}\right|<1 \cdot e^{-5}$, where

$$
\epsilon_{t, v}=\hat{J}_{t}-\rho \hat{J}(v, t)+\hat{J}_{v}\left(v\left[\left(\hat{\boldsymbol{\theta}}_{t}^{o p t}\right)^{\prime}(\boldsymbol{\mu}-\boldsymbol{r})+r\right]\right)+\frac{1}{2} v^{2}\left\|\left(\hat{\boldsymbol{\theta}}_{t}^{o p t}\right)^{\prime} \boldsymbol{\sigma}\right\|^{2} \hat{J}_{v v}+U(v) .
$$

\section{Computation of the value function}

Let $\widehat{\boldsymbol{\theta}}_{t}^{\text {opt }}(v, t)$ be the investment strategy from the above numerical algorithm. If we substitute $\widehat{\boldsymbol{\theta}}_{t}^{\text {opt }}(v, t)$ into the HJB equation (6), it is found that the value function $\hat{J}(v, t)$ is the solution of the partial differential equation

$$
\begin{aligned}
0=J_{t}(v, t)-\rho J(v, t)+J_{v}(v, t)\left(v \left[\left(\hat{\theta}_{t}^{o p t}(v, t)\right)^{\prime}\right.\right. & (\mu-r)+r]) \\
& +\frac{1}{2} v^{2}\left\|\left(\hat{\theta}_{t}^{o p t}(v, t)\right)^{\prime} \sigma\right\|^{2} J_{v v}(v, t)+U(v),
\end{aligned}
$$

where $J_{t}(v, t), J_{v}(v, t), J_{v v}(v, t)$ denote the first-order derivatives of the value function with respect to $t$ and $v$, and the second-order derivative with respect to $v$, respectively.

Recall that the value function $J(v, t)$ solves the HJB equation for all possible values of $v$ and all $t \in[0, T]$ and satisfies the terminal condition

$$
\begin{aligned}
J\left(V_{T}, T\right) & =U\left(V_{T}\right)=K V_{T}-(1-K)\left(V_{T}-\psi\right)^{2} \\
& =-V_{T}^{2}(1-K)+V_{T}(K+2 \psi(1-K))-(1-K) \psi^{2} .
\end{aligned}
$$

We therefore guess, for $t<T$, that $J(v, t)=a(t) v^{2}+b(t) v+c(t)$ with auxiliary functions $a, b$ and $c$. We note here that as a consequence of the imposition of the constraint, the auxiliary functions $a, b$ and $c$ will generally depend on $v$ and $t$. Borrowing from an idea of Yiu [15], if we neglect the derivatives of these auxiliary functions with respect to $v$, then the partial derivatives of $J$ are $J_{v}=2 a(v, t) v+b(v, t), J_{v v}=2 a(v, t)$ and $J_{t}=a^{\prime}(v, t) v^{2}+b^{\prime}(v, t) v+c^{\prime}(v, t)$. Introducing these derivatives into (7) and regrouping the terms in $v^{2}, v$ and independent of $v$, gives three different ordinary differential equations for $a, b$ and $c$ respectively. 
Now substituting the partial derivatives into (7) we obtain

$$
\begin{aligned}
0=v^{2}\left(a^{\prime}(v, t)-\right. & \rho a(v, t)-(1-K)+2 a(v, t)\left[\left(\hat{\theta}_{t}^{o p t}(v, t)\right)^{\prime}(\mu-r)+r\right] \\
+a(v, t) & \left.\left\|\left(\hat{\theta}_{t}^{o p t}(v, t)\right)^{\prime} \sigma\right\|^{2}\right)+v\left(b^{\prime}(v, t)+\rho b(v, t)+K+2(1-K) \psi\right. \\
& \left.+b(v, t)\left[\left(\hat{\theta}_{t}^{o p t}(v, t)\right)^{\prime}(\mu-r)+r\right]\right)+c^{\prime}(v, t)+\rho c(v, t)-(1-K) \psi^{2} .
\end{aligned}
$$

Since (8) must hold for all $v$ and $t$, we obtain the system of ordinary differential equations

$$
\begin{aligned}
& a^{\prime}(v, t)=\rho a(v, t)+(1-K)-2 a(v, t)\left[\left(\hat{\boldsymbol{\theta}}_{t}^{o p t}(v, t)\right)^{\prime}(\boldsymbol{\mu}-\boldsymbol{r})+r\right]-a(v, t)\left\|\left(\hat{\boldsymbol{\theta}}_{t}^{o p t}(v, t)\right)^{\prime} \boldsymbol{\sigma}\right\|^{2} \\
& b^{\prime}(v, t)=-\rho b(v, t)-K-2(1-K) \psi-b(v, t)\left[\left(\hat{\boldsymbol{\theta}}_{t}^{o p t}(v, t)\right)^{\prime}(\boldsymbol{\mu}-\boldsymbol{r})+r\right] \\
& c^{\prime}(v, t)=-\rho c(v, t)+(1-K) \psi^{2}
\end{aligned}
$$

with terminal conditions $a(v, T)=-(1-K), b(v, T)=K+2(1-K) \psi$ and $c(v, T)=$ $-(1-K) \psi^{2}$. The functions $a, b$ and $c$ may be computed numerically by means of the Euler-Cauchy method (see, for example, Isaacson and Keller [8]). We use a hat to denote a result obtained by menas of numerical integration. Then

$$
\begin{array}{ll}
\hat{a}(v, t-\Delta t)=\hat{a}(v, t)-a^{\prime}(v, t) \Delta t, & \hat{a}(v, T)=-(1-K), \\
\hat{b}(v, t-\Delta t)=\hat{b}(v, t)-b^{\prime}(v, t) \Delta t, & \hat{b}(v, T)=K+2(1-K) \psi, \\
\hat{c}(v, t-\Delta t)=\hat{c}(v, t)-c^{\prime}(v, t) \Delta t, & \hat{c}(v, T)=-(1-K) \psi^{2},
\end{array}
$$

where $\Delta t$ is a sufficiently small time step. The precision of the solution is a function of the step length of integration $\Delta t$ and is computed via the Bellman equation (7). If we

subdivide the domain of computation into a grid of $N_{v} \times N_{t}$ mesh points, the error at time $t$ from a wealth level $v$ with $v \in\left[0, \Delta v, \ldots, N_{v} \Delta v\right]$ and $t \in\left[0, \Delta t, \ldots, N_{t} \Delta t\right]$ is

$$
\epsilon_{t, v}=\hat{J}_{t}-\rho \hat{J}(v, t)+\hat{J}_{v}\left(v\left[\left(\hat{\theta}_{t}^{o p t}\right)^{\prime}(\mu-r)+r\right]\right)+\frac{1}{2} v^{2}\left\|\left(\hat{\theta}_{t}^{o p t}\right)^{\prime} \sigma\right\|^{2} \hat{J}_{v v}+U(v),
$$

where

$$
\begin{aligned}
\hat{J} & =\hat{a}(v, t) v^{2}+\hat{b}(v, t) v+\hat{c}(v, t), \\
\hat{J}_{v} & =2 \hat{a}(v, t) v+\hat{b}(v, t), \\
\hat{J}_{v v} & =2 \hat{a}(v, t), \\
\hat{J}_{t} & =\hat{a}^{\prime}(v, t) v^{2}+\hat{b}^{\prime}(v, t) v+\hat{c}^{\prime}(v, t)
\end{aligned}
$$

and $\hat{\boldsymbol{\theta}}_{t}^{\text {opt }}(v, t)$ is obtained via the numerical algorithm detailed in Section 6 above.

\section{Simulation results and discussion}

We implemented the algorithm in Section 6 in MATLAB 7.0 in order to test our model on a personal computer with an Intel Pentium IV processor. We assume that $n=2$. That is, the market is composed of two risky stocks and a risk-free bond. Table 1 shows the parameters for the portfolio optimization problem and the underlying Black-Scholes model of the financial market. We achieved convergence in under 300 seconds after three iterations. 


\begin{tabular}{ll}
\hline Parameter & Value \\
\hline Stock $\left(S^{1}\right)$ & $\mu=4 \%, \sigma^{11}=5 \%, \sigma^{12}=5 \%$ \\
Stock $\left(S^{2}\right)$ & $\mu=6 \%, \sigma^{21}=5 \%, \sigma^{22}=20 \%$ \\
Bond $\left(S^{0}\right)$ & $r=3 \%$ \\
Investment horizon & $t \in[0,1]$ \\
State of wealth & $v \in[0,20]$ \\
Shortfall probability & $\alpha=1 \%$ \\
Value-at-Risk horizon & $\Delta t=\frac{1}{48} \approx 7$ days \\
No. of wealth mesh points & $N_{v}=81$ \\
Mesh size for wealth & $\Delta v=\frac{20}{80}=0.25$ \\
Quadratic utility function & $U(v)=K v-(1-K)(v-\psi)^{2}, K=0.6, \psi=5$. \\
Discounting factor & $\rho=0.3$ \\
\hline
\end{tabular}

Table 1: Parameters for portfolio optimization problem.

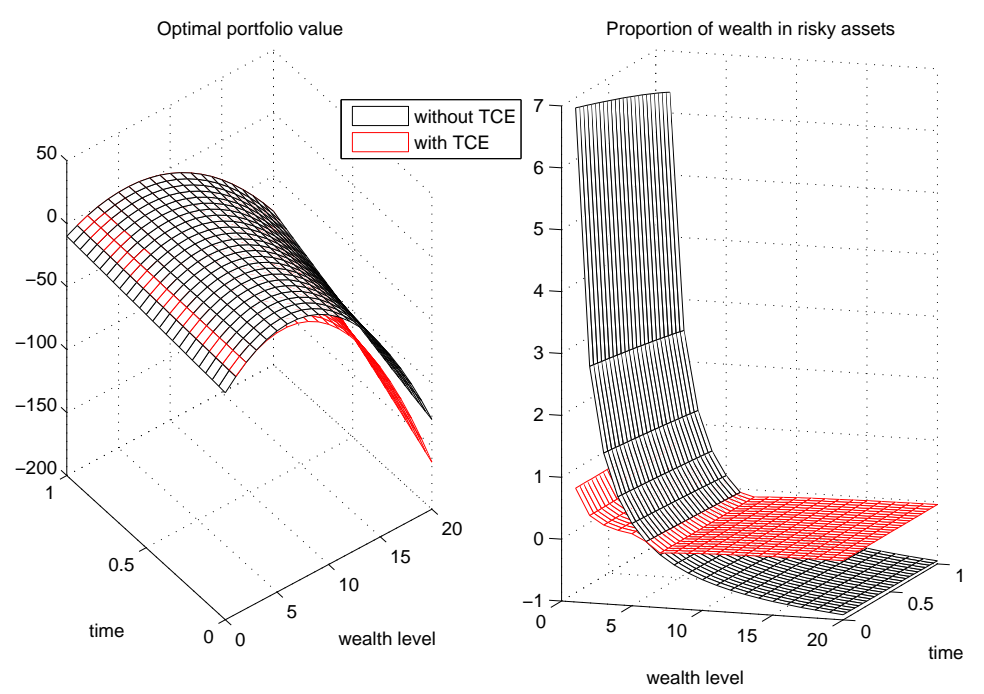

Figure 1: Effect of the TCE constraint on the proportion of risky investment when the benchmark is conditional expectation of wealth.

\subsection{Static constraint}

Suppose that the Tail Conditional Expectation of the wealth surplus $\left(V_{t+\Delta t}-\Upsilon_{t+\Delta t}\right)$ with respect to the benchmark $\Upsilon_{t+\Delta t}$ satisfies $T C E_{t}^{\alpha}\left(V_{t+\Delta t}-\Upsilon_{t+\Delta t}\right) \leq 0.05$. That is, the TCE is re-evaluated at each discrete time step (TCE horizon) $\Delta t$ and kept below the upper bound $\varepsilon\left(V_{t}, t\right)=0.05$, by making use of conditioning information. A plot of the investment in risky assets is shown in the right-hand panels of Figures 1 and 2 with and without the TCE constraint against the possible wealth realization at different times. The left-hand panels show the corresponding value functions.

In Figure 1 the shortfall benchmark is taken to be the conditional expected wealth $\Upsilon_{t+\Delta t}=$ $\mathbb{E}_{t}\left\{V_{t+\Delta t}\right\}=V_{t} \exp \left[\left(\theta_{t}^{\prime}(\mu-r)+r\right) \Delta t\right]$ at each TCE horizon before the terminal date $T$, while in Figure 2 it is the investment in the risk-free bond $\Upsilon_{t+\Delta t}=V_{t} e^{r \Delta t}$.

We observe that as the wealth level increases, the amount of wealth invested in stocks diminishes. This results from the "increasing absolute risk aversion" that characterizes 


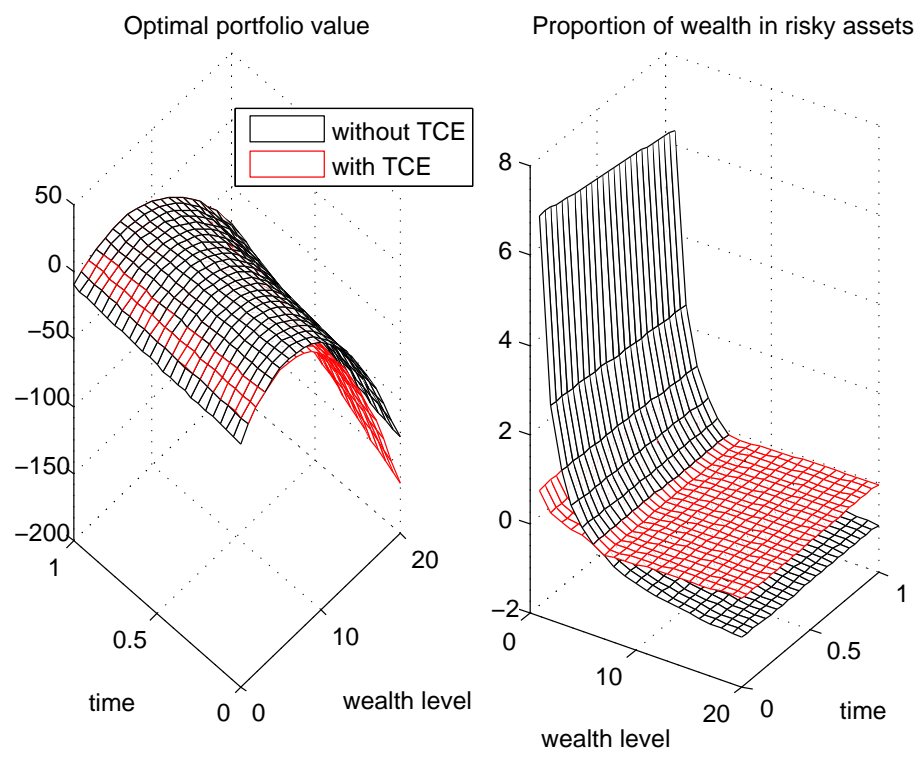

Figure 2: Effect of the TCE constraint on proportion of risky investment when the benchmark is investment in the bond.

the quadratic nature of the utility function. Furthermore, it may be observed that when the wealth is smaller than the utility target $\psi=5$ of the quadratic utility function, the optimal solution consists of increasing the position of stocks. The exposure to the market is, however, limited by the VaR constraint which bounds the amount invested in stocks. On the other hand, as wealth increases above $\psi$, the position in stocks is reduced, but again limited by the VaR constraint.

This is a rather counter-intuitive investment strategy, but results directly from the quadratic nature of the utility function, whereby utility rises up to the satiation level $\psi$ and diminishes thereafter. One would expect that as the wealth level falls the strategy might shift into lower-risk assets to protect one's position. The strategy we have found does the opposite. As mentioned, the reason for this is because of the quadratic form of the objective function. This function, in a sense, defines an ideal wealth level $\psi$. If wealth is below this level, then one invests in high-return, high-risk assets to increase the chance of returning to the ideal level quickly. Conversely, if the wealth level is too high, then one is prepared to invest in an inefficient low-return investment strategy in order to return to the ideal level. Owing to this shortcoming of quadratic utility, one could instead consider applying power utility with its more intuitive constant relative risk aversion property (see, for example, Akume and Mbakop [1]).

The value function of the constrained problem is identical to that of the unconstrained one when the Lagrange multipliers are zero (the optimal constrained portfolio follows the unconstrained one), whereas it is inferior when the constraint is active (allocation to risky assets is controlled).

In Figure 3, the constrained and unconstrained TCE values are shown in the wealth/time space. The constrained and unconstrained strategies coincide in the area where the lighter 


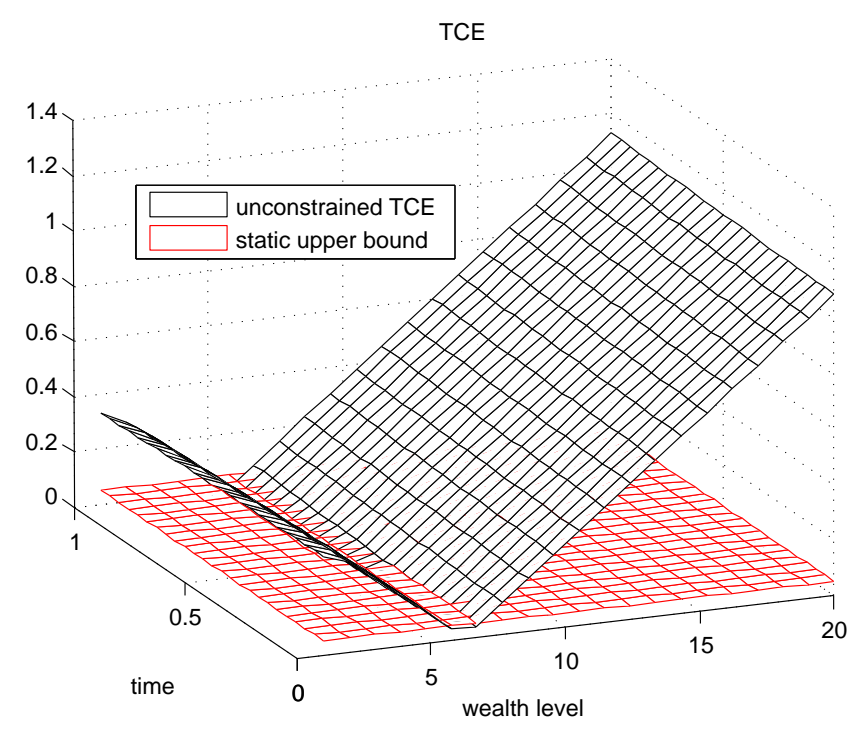

Figure 3: TCE when the benchmark is the conditional expected wealth, plotted against wealth at various times of the investment horizon.

surface (TCE limit) lies above the dark surface.

\subsection{Dynamic constraint}

So far, we have constrained the risk measure from above by a static constraint $\varepsilon\left(V_{t}, t\right)=$ 0.05. We next attempt to change the risk limit $\varepsilon\left(V_{t}, t\right)=-0.005\left(n_{v} \Delta v-V_{t}\right)+0.2$ (see Figure 5) dynamically, where $n_{v}$ denotes the number of discrete wealth intervals and where $\Delta v$ denotes the mesh size, in order to assertain th impact that this approach has on the optimal portfolio choice.

This conforms to what accurs in practice, whereby successful traders typically see their risk limit increased. Thus, the larger the wealth, the larger the risk bound.

Figure 4 shows that even with this dynamic bound, limiting the TCE keeps the investment in risky assets in check.

The constrained and unconstrained TCE values are compared in Figure 5 for the dynamic constraint. Note how the limiting TCE ceiling lies at an increasing slope to the horizontal plane, since it is no longer static as before.

\section{Concluding remarks}

Using a quadratic utility function, we investigated in this paper how a bound imposed on TCE affects the optimal portfolio choice. We used dynamic wealth benchmarks conditional expected wealth and investment in risk-free bonds - whereby the TCE was reevaluated at short intervals along the investment horizon. We deduce from our numerical results that the constraint is able to control risky investment. 


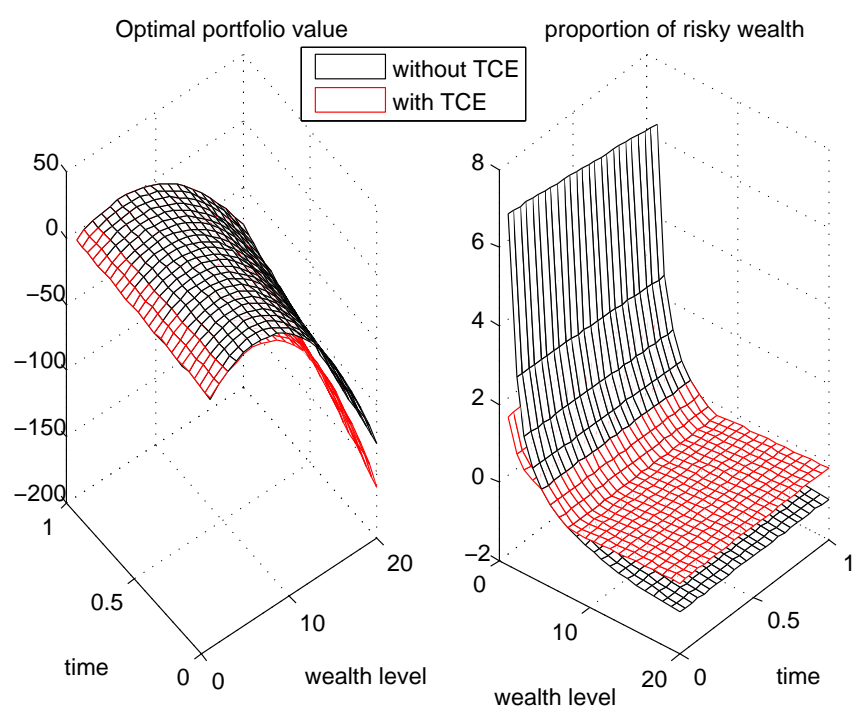

Figure 4: Effect of dynamic TCE constraint when the benchmark is conditional expectation of wealth.

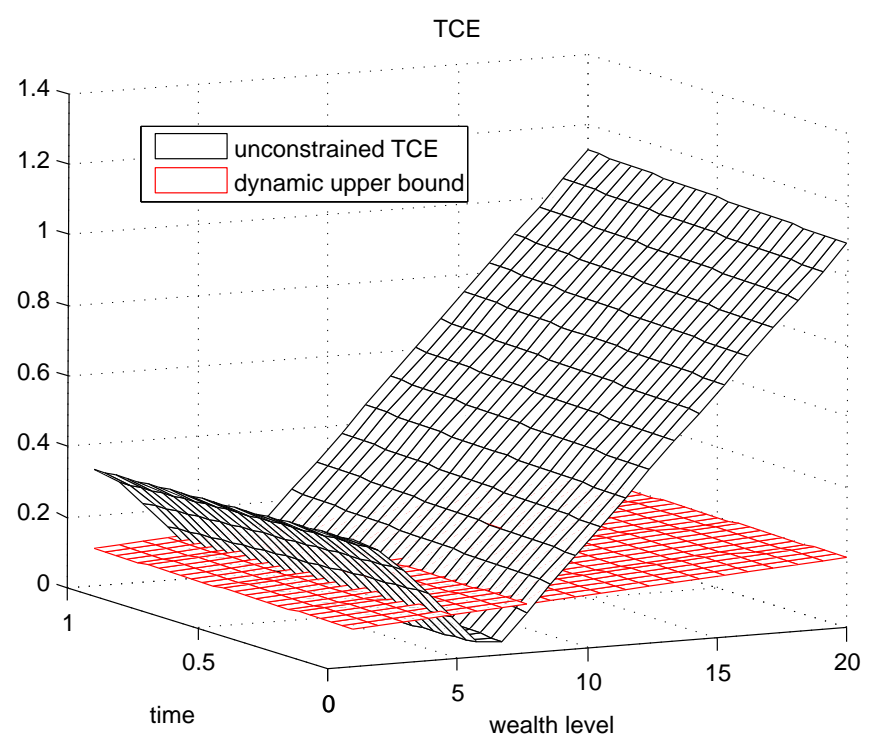

Figure 5: Dynamic TCE upper bound versus the unconstrained TCE when the benchmark is the conditional expected wealth, plotted against wealth at various times of the investment horizon.

\section{Acknowledgement}

The first author was supported by the African Economic Research Consortium (AERC) under grant number TT07513, at the University of Yaounde I. 


\section{References}

[1] Aкume D \& Mвакор GM, 2008, Optimal dynamic portfolios under a tail conditional expectation constraint, Studia Universitas Babes-Bolyai Mathematica, LIII(4), pp. 3-21.

[2] Artzner P, Delbaen F, Eber JM \& Heath D, 1997, Thinking coherently, Risk, 10, pp. 68-71.

[3] Artzner P, Delbaen F, Eber, JM \& Heath D, 1999, Coherent measures of risk, Mathematical Finance, 9(3), pp. 203-228.

[4] Cuoco D, He H \& Isaenko S, 2008, Optimal dynamic trading strategies with risk limits, Operations Research, 56(2), pp. 358-368.

[5] Fleming WH \& Rishel RW, 1975, Deterministic and stochastic optimal control, Springer Verlag, Berlin.

[6] Haberman S \& Sung JH, 1994, Dynamic approaches to pension funding, Insurance Mathematics and Economics, 15, pp. 151-162.

[7] He H \& PEARson N, 1991, Consumption and portfolio policies with incomplete markets and shortsale constraints: The finite dimensional case, Mathematical Finance, 1, pp. 1-10.

[8] Isaacson E \& Keller HB, 1994, Analysis of numerical methods, Dover Publications, New York $(\mathrm{NY})$.

[9] JoRion P, 1997, Value at risk: The new benchmark for controlling derivative risk, McGraw-Hill Publishers, Blacklick (OH).

[10] Korn R, 1997, Optimal portfolios, World Scientific, Singapore.

[11] Markowitz HM, 1952, Portfolio selection, The Journal of Finance, 7(1), pp. 77-91.

[12] McNeil AJ, Frey R \& Embrechts P, 2006, Quantitative risk management: Concepts, techniques and tools, Princeton University Press, Princeton (NJ).

[13] Nocedal J \& Wright S, 1999, Martingale methods in financial modeling, Springer Verlag, Heidelberg.

[14] Rockafellar RT \& Uryasev S, 2002, Conditional value-at-risk for general loss distributions, Journal of Banking and Finance, 26(7), pp. 1443-1471.

[15] YIU KFC, 2004, Optimal portfolios under a value at risk constraint, Journal of Economic Dynamics and Control, 28, pp. 1317-1334. 